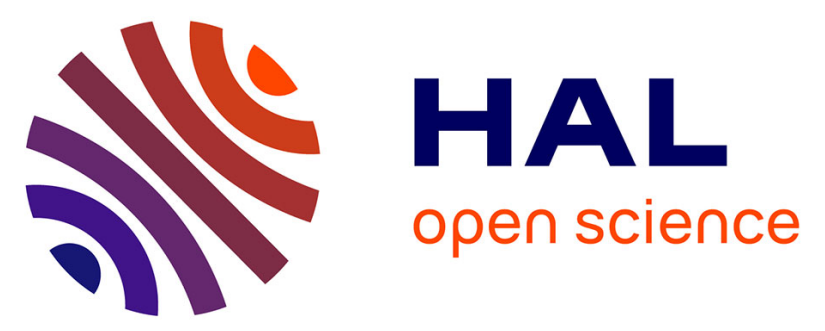

\title{
Helicene-Based Ligands Enable Strong Magneto-Chiral Dichroism in a Chiral Ytterbium Complex
}

\author{
Matteo Atzori, Kais Dhbaibi, Haiet Douib, Maxime Grasser, Vincent Dorcet, \\ Ivan Breslavetz, Kévin Paillot, Olivier Cador, Rikken G.L.J.A., Boris Le \\ Guennic, et al.
}

\section{To cite this version:}

Matteo Atzori, Kais Dhbaibi, Haiet Douib, Maxime Grasser, Vincent Dorcet, et al.. Helicene-Based Ligands Enable Strong Magneto-Chiral Dichroism in a Chiral Ytterbium Complex. Journal of the American Chemical Society, 2021, 143 (7), pp.2671-2675. 10.1021/jacs.0c13180 . hal-03156581

\section{HAL Id: hal-03156581 https://hal.science/hal-03156581}

Submitted on 13 Apr 2021

HAL is a multi-disciplinary open access archive for the deposit and dissemination of scientific research documents, whether they are published or not. The documents may come from teaching and research institutions in France or abroad, or from public or private research centers.
L'archive ouverte pluridisciplinaire HAL, est destinée au dépôt et à la diffusion de documents scientifiques de niveau recherche, publiés ou non, émanant des établissements d'enseignement et de recherche français ou étrangers, des laboratoires publics ou privés. 


\title{
Helicene-based Ligands Enable Strong Magneto-Chiral Dichro- ism in a Chiral Ytterbium Complex
}

\author{
Matteo Atzori, ${ }^{*}{ }^{\dagger}$ Kais Dhbaibi, ${ }^{\ddagger}$ Haiet Douib, ${ }^{\ddagger}$ Maxime Grasser, $^{\ddagger}$ Vincent Dorcet, ${ }^{\ddagger}$ Ivan Breslavetz, $^{\dagger}$ \\ Kévin Paillot, ${ }^{\dagger}$ Olivier Cador, ${ }^{\ddagger}$ Geert L. J. A. Rikken, ${ }^{\dagger}$ Boris Le Guennic, ${ }^{\ddagger}$ Jeanne Crassous, ${ }^{*, \ddagger}$ Fabrice \\ Pointillart, ${ }^{* \neq}$ and Cyrille Train ${ }^{\dagger}$ \\ $\dagger \quad$ Laboratoire National des Champs Magnétiques Intenses (LNCMI), Univ. Grenoble Alpes, INSA Toulouse, Univ. \\ Toulouse Paul Sabatier, EMFL, CNRS, Grenoble, France \\ ‡ Univ Rennes, CNRS, ISCR (Institut des Sciences Chimiques de Rennes) - UMR 6226, F-35000 Rennes, France
}

Supporting Information Placeholder

\begin{abstract}
Here we report the first experimental observation of magneto-chiral dichroism (MChD) detected through light absorption in an enantiopure lanthanide complex. The $P$ and $M$ enantiomers of $\left[\mathrm{Yb}^{\mathrm{III}}((X)-\mathbf{L})(h f a c)_{3}\right](X=P, M ; \mathbf{L}=3$-(2-pyridyl)4-aza[6]-helicene; $h f a c=1,1,1,5,5,5$-hexafluoro-acetylacetonate), where the chirality is held by the helicene-based ligand, were studied in the near infrared spectral window. When irradiated with unpolarized light in a magnetic field, these chiral complexes exhibit a strong MChD signal ( $\mathrm{g}_{\mathrm{MChD}}$ ca. $0.12 \mathrm{~T}^{-1}$ ) associated to the ${ }^{2} \mathrm{~F}_{5 / 2} \leftarrow{ }^{2} \mathrm{~F}_{7 / 2}$ electronic transition of $\mathrm{Yb}^{\text {III }}$. The low temperature absorption and MChD spectra reveal a fine structure associated with crystal field splitting and vibronic coupling. The temperature dependence of the main dichroic signal detected up to $150 \mathrm{~K}$ allowed to disentangle for the first time the two main microscopic contributions to the dichroic signal predicted by the MChD theory. These findings pave the way towards probing MChD in chiral lanthanide-based single-molecule magnets.
\end{abstract}

Magneto-Chiral Dichroism (MChD) represents a fascinating manifestation of light-matter interaction in chiral magnetized systems. ${ }^{1}$ It features a modification of the absorption or emission of unpolarized light that depends on the relative orientation of the magnetic field $(\boldsymbol{B})$, the direction of light propagation $(\boldsymbol{k})$, and the absolute configuration of the system. ${ }^{2,3}$ This phenomenon, predicted in 1984 and first observed in 1997, has been recently proposed for optical data readout. ${ }^{4-6}$ Its reverse effect, that is, the generation of enantiomeric enrichments by irradiating a racemic mixture of enantiomers with unpolarized light in magnetic field, has been observed and proposed as a possible mechanism for the origin of life homochirality. ${ }^{1,3}$ For both topics, it is crucial to identify the electronic and structural features that drive $\mathrm{MChD}$, such as the nature of the metal ion and the type of chirality. The microscopic theory of MChD identifies several physical mechanisms that generate $\mathrm{MChD},{ }^{2}$ each having a particular lineshape and temperature dependence, but so far this theory has not been verified experimentally. Identifying these mechanisms will help to promote our understanding of MChD.

MChD has been studied on a limited number of chiral molecular systems. ${ }^{7,8}$ Renewed interest in this phenomenon has allowed its detection in $2 \mathrm{D}$ chiral perovskites, ${ }^{9}$ and in a $2 \mathrm{D}$ molecular ferrimagnet at temperatures as high as $40 \mathrm{~K} .{ }^{10}$ More recently, MChD investigations on a molecular magnetic helix based on Jahn-Teller elongated $\mathrm{Mn}^{\mathrm{III}}$ ions have revealed the crucial role of spin-orbit coupling into MChD intensity when probed with visible light, ${ }^{11}$ originally underlined with hard X-rays. ${ }^{12}$

These recent results call for a study of MChD in chiral lanthanide systems because they possess a stronger spin-orbit coupling than first row transition metals and allow for impressive single-molecule magnet (SMM) properties. ${ }^{13,14}$ Indeed, long-range ordered chiral magnets ${ }^{15}$ or SMMs ${ }^{16}$ showing strong MChD can be envisioned for optical readout of magnetic data with unpolarized light. This consists in distinguishing two orientations of the magnetization by measuring the difference in absorption, reflection or emission of light. Due to the weak absorption coefficients of $f-f$ electronic transitions, the scarce studies on MChD in lanthanide complexes were performed investigating their light emission properties, ${ }^{1,17}$ while studies focused on light absorption are missing.

Lanthanide complexes are often associated with high coordination numbers, low local symmetries, and configurational lability, which makes difficult to control their stereochemistry. A more versatile approach consists of introducing the chirality on the coordinating ligand. Recent studies on transition metal complexes have demonstrated that second coordination sphere chiral features enable MChD without the need of a strongly chiral environment surrounding the metal. ${ }^{9-11,18}$ This opens the door towards the design of a much larger variety of chiral complexes showing MChD. In parallel, the incorporation of inherently chiral ligands into organometallic architectures has shown unique properties resulting from the strong chiroptical response of the ligand and the intrinsic properties of the metal. ${ }^{19}$

For this first study of MChD through NIR light absorption on lanthanide complexes, we have prepared enantiopure $\mathrm{Yb}^{\mathrm{III}}$ complexes formulated as $\left[\mathrm{Yb}^{\mathrm{III}}((X)-\mathbf{L})(h f a c)_{3}\right](X=P, M ; \mathbf{L}=3-(2-\mathrm{py}-$ ridyl)-4-aza[6]-helicene; $h f a c=1,1,1,5,5,5$-hexafluoro-acetylacetonate) (1-(P), 1-(M)). In these complexes, the helicene-based chiral bipyridine ligand has an intense chiro-optical activity demonstrated by its strong natural circular dichroism (Figure S1), and the lanthanide ion has one of the simplest electronic configurations in the lanthanide series. By featuring only one excited multiplet $\left({ }^{2} \mathrm{~F}_{5 / 2}\right)$, it admits only one well-defined magnetic dipole allowed $(|\Delta J|=1)$ electronic transition, ${ }^{2} \mathrm{~F}_{5 / 2} \leftarrow{ }^{2} \mathrm{~F}_{7 / 2}$. 
Complexes 1-(P) and 1-(M) are obtained by reaction of enantiopure (P)- and (M)-L with $\left[\mathrm{Yb}^{\mathrm{III}}(h f a c)_{3}\left(\mathrm{H}_{2} \mathrm{O}\right)_{2}\right]$ in $\mathrm{CH}_{2} \mathrm{Cl}_{2}$ (see SI). ${ }^{16,20} \mathbf{1}$ crystallizes in the orthorhombic $P 22_{1} 2_{1} 2_{1}$ space group (Table S1) and is isostructural to its Dy ${ }^{\mathrm{III}}$ analogue. ${ }^{16}$ It features one crystallographically independent molecule in the asymmetric unit where the chirality on the ligand induces a helical chirality at the lanthanide center ( $\Delta$-type for 1-(P), $\Lambda$-type for 1-(M)) (Figure 1 and S2). The crystal structure shows $\pi-\pi$ contacts between helicene aromatic rings of adjacent molecules that develop along the $a$ axis (Figure $\mathrm{S} 3$ ).

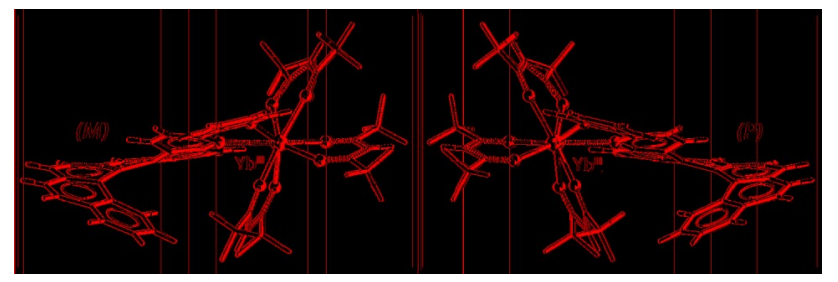

Figure 1. Molecular structures of 1-(M) and 1-(P).

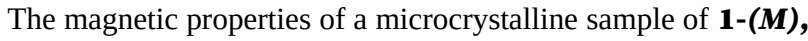
whose phase purity was checked by powder X-ray diffraction analysis (Figure S4), were investigated. The molar magnetic susceptibility times the temperature provides at room temperature a value of $2.35 \mathrm{~cm}^{3} \mathrm{~K} \mathrm{~mol}^{-1}$, which is consistent with the expected value $\left(2.57 \mathrm{~cm}^{3} \mathrm{~K} \mathrm{~mol}^{-1}\right)$ assuming a $J=7 / 2\left(S=1 / 2, L=3, \mathbf{g}_{\mathrm{J}}=\right.$ $8 / 7$ ) ground state (Figure S5). The magnetization at $T=2.0 \mathrm{~K}$ almost saturates at $7.0 \mathrm{~T}$ with a value of ca. $1.5 \mu_{\mathrm{B}} \mathrm{mol}^{-1}$, also in agreement with the expected value $\left(1.6 \mu_{\mathrm{B}} \mathrm{mol}^{-1}\right)$ (Figure S6). Alternating current susceptibility measurements reveal a slow magnetic relaxation at high frequency when a small magnetic field is applied (Figure S7).

To get insights in the electronic transitions of $\mathbf{1}$, enantiopure single crystals of 1-(P) and 1-(M), were used to record absorption spectra in transmission mode with unpolarized light propagating perpendicularly to the [011] crystallographic face and the $a$ axis (Figure S8) as a function of the temperature (290-4.0 K). At room temperature, the electronic spectrum shows two main features: an intense shoulder associated to the lowest energy absorption band of the ligand $\mathbf{L}$, in agreement with measurements performed in solution (Figure S1), and a series of overlapping absorption bands in the 920-990 $\mathrm{nm}$ range, with a sharp contribution at $\lambda=980 \mathrm{~nm}$ (Figures S9, S10). This is assigned to the ${ }^{2} \mathrm{~F}_{5 / 2} \leftarrow$ ${ }^{2} \mathrm{~F}_{7 / 2}$ electronic transition of $\mathrm{Yb}^{\mathrm{III}} .{ }^{21}$

At $4.0 \mathrm{~K}$ the $\mathrm{Yb}^{\mathrm{III}}$ absorption becomes more structured, evidencing four main contributions at $\lambda=933,940,958,980 \mathrm{~nm}$ (Figure 2a). Taking into account the crystal field splitting of the ${ }^{2} \mathrm{~F}_{7 / 2}$ ground and ${ }^{2} \mathrm{~F}_{5 / 2}$ excited multiplets in $(2 J+1) / 2$ Kramers doublets separated by hundreds of $\mathrm{cm}^{-1}$ (Figure 2c), at $4.0 \mathrm{~K}$ only the lowest energy sublevel of the ground multiplet is thermally populated $\left(k_{B} T=2.78 \mathrm{~cm}^{-1}\right)$, thus, only three transitions should be observed (Figure 2c).

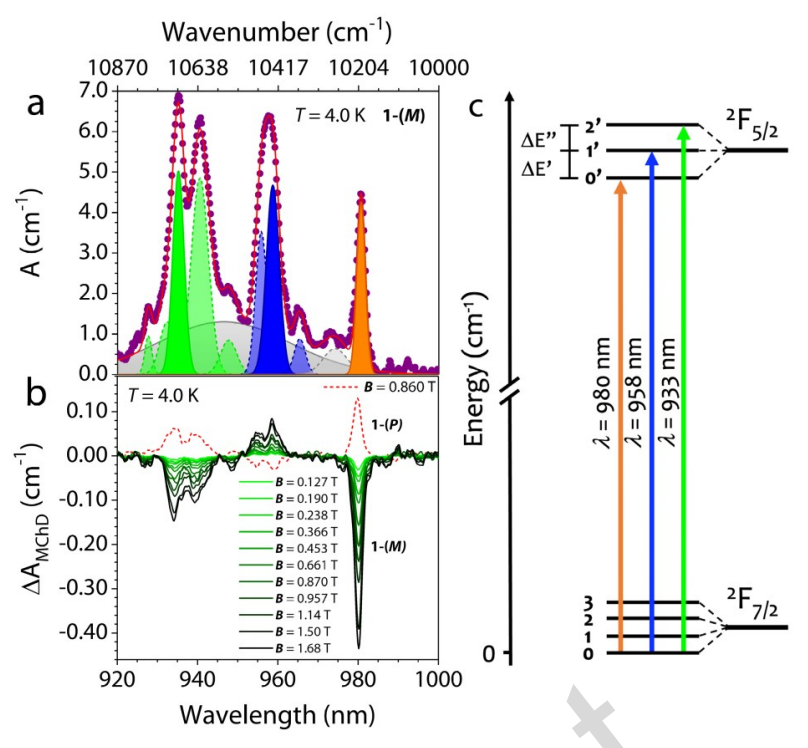

Figure 2. Absorption spectrum for a single crystal of 1-(M) with spectral deconvolution analysis (a), and field dependence of $\Delta \mathrm{A}_{\mathrm{M} \text { - }}$ ChD (b). Schematic representation of the energy level diagram (c). The colored sub-peaks represent the vibronic contribution to the three electronic transitions between Kramers doublets, as deduced from the sign of the MChD signals (see below).

From further investigation (see below), the two high energy signals are believed to correspond to a unique electronic transition between Kramers doublets of the ground and excited states (green arrow in Figure 2c). The difference in energy between the three main transitions, are $234 \mathrm{~cm}^{-1}$ and $280 \mathrm{~cm}^{-1}$, which fall well within the typical range for crystal field splittings of $J$ multiplets of lanthanide ions. These values are in agreement with those obtained by CAS(13,7)PT2/SI-SO calculations $\left(250 \mathrm{~cm}^{-1}, 254 \mathrm{~cm}^{-1}\right)$, which allowed to determine the composition and the order in energy of the Kramers doublets imposed by the $\mathrm{Yb}^{\mathrm{III}}$ magnetic anisotropy (Tables S2, S3).

The discrepancy between the number of expected and observed transitions is rather puzzling and calls for further discussion. Lowering temperature could lead to a symmetry lowering of the sys tem, but this possibility would not allow further crystal field splitting of the energy levels. Hot bands that are extra transitions from thermally populated excited states, are ruled out by the thermal population distribution at $4.0 \mathrm{~K}$. The only possibility appears to be the vibronic coupling between the described electronic transitions and low energy vibrational modes. To validate this possibility, Raman spectra were recorded on a single crystal of $\mathbf{1 - ( P )}$ by using a $\lambda=781 \mathrm{~nm}$ laser to avoid that the laser excitation activates the luminescence of the ligand. ${ }^{20}$ The typical D- and G-band of the helicene moiety, that are associated to $\mathrm{C}=\mathrm{C}$ stretching, are clearly observed (Figure S11). ${ }^{22,23}$ Moreover, the Raman spectrum shows a multitude of signals (Figure S12) in the energy range of the finesplitting observed in the electronic spectrum, which is a strong indication of its vibronic origin.

MChD measurements were performed at $T=4.0 \mathrm{~K}$ with an alternating $(\Omega=1.5 \mathrm{~Hz}$ ) magnetic field $\boldsymbol{B}$ applied along $\boldsymbol{k}$ in the $0.0-1.86$ T range. Figure $2 \mathrm{~b}$ shows the MChD spectra for the two enantiomers at the intermediate magnetic field $\boldsymbol{B}=0.860 \mathrm{~T}$ while the full $\boldsymbol{B}$ dependence is shown for $\mathbf{1 - ( M )}$. Strong MChD signals originating from the difference in the absorption of the ${ }^{2} \mathrm{~F}_{5 / 2} \leftarrow$ ${ }^{2} \mathrm{~F}_{7 / 2}$ transition split by the crystal field and the vibronic coupling are observed (Figure 2b), with equal intensities and opposite signs for the two enantiomers, resulting in mirrored spectra (Figure 
S13). The main MChD signal is associated to the lowest energy 0' $\leftarrow 0$ transition. Since they are more affected by the vibronic substructure, the 1 ' $\leftarrow 0$ and 2 ' $\leftarrow 0$ transitions have lower intensities and provide broader MChD signals. An interesting feature of the $\mathrm{MChD}$ spectra is the alternation of its sign with the nature of the excited Kramers doublets. The similitude between the shape of the MChD and the rare NCD spectra reported for chiral $\mathrm{Yb}^{\mathrm{III}}$ complexes, ${ }^{21,24}$ highlights that to fully unravel the link between these phenomena, MChD, NCD and MCD measurements in the same experimental conditions are mandatory, although they remain technically challenging. The asymmetric factor $\mathrm{g}_{\mathrm{MChD}}$ (see SI), which evaluate the portion of absorption or emission that is af fected by the magnetic field, is ca. $0.12 \mathrm{~T}^{-1}$, thus comparable to that of a chiral $\mathrm{Tb}^{\mathrm{III}}$ complex $\left(0.06 \mathrm{~T}^{-1}\right)$ investigated through light emission. ${ }^{17}$

Thanks to its high intensity, the MChD signal at $980 \mathrm{~nm}$ can be detected up to $150 \mathrm{~K}$ even when a low magnetic field is applied. MChD measurements were thus performed at $\boldsymbol{B}= \pm 0.860 \mathrm{~T}$ to study the temperature dependence (4.0-150 K) of $\Delta \mathrm{A}_{\mathrm{MChD}}$ for both enantiomers (Figure S14). The temperature and the magnetic field dependence of $\Delta \mathrm{A}_{\mathrm{MChD}}(\lambda=980 \mathrm{~nm})$ is compared in Figure 3 with magnetization data recorded in the same conditions on a microcrystalline sample of 1-(M).
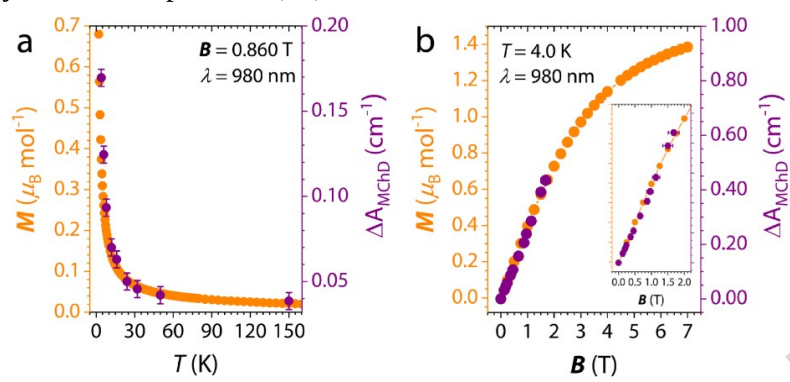

Figure 3. Temperature (a) and magnetic field (b) dependence of the $\Delta \mathrm{A}_{\mathrm{MChD}}$ signal $(\lambda=980 \mathrm{~nm})$ of $\mathbf{1}-(\boldsymbol{M})$ compared to magnetization data.

Figure 3 shows that $\Delta \mathrm{A}_{\mathrm{MChD}}$ accurately follows the magnetization of the sample, as predicted by the theory, and highlights that this dichroic signal could be further enhanced. Most interestingly, the extensive temperature range explorable for this system has allowed to observe an unprecedented feature in the band-shape of the main MChD signal. At low temperatures, it has an absorptionlike line-shape. When the temperature increases, the intensity becomes lower as a result of the lower magnetization (see above), but more importantly, the line-shape changes, becoming of derivative-type (Figure 4a).

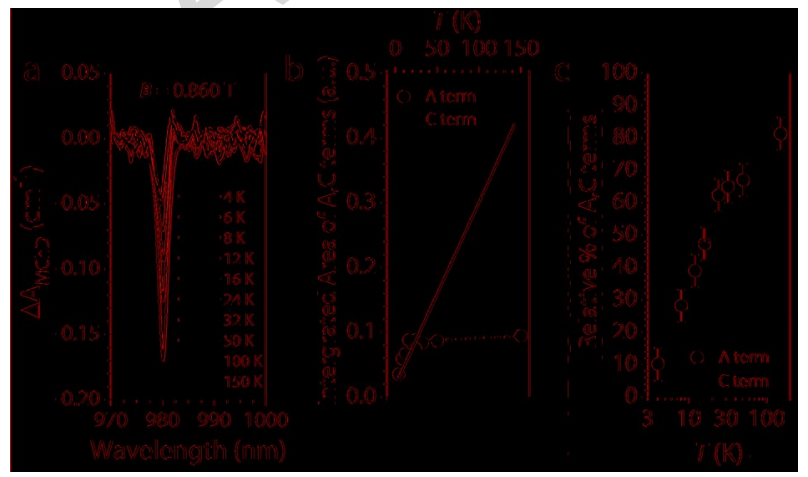

Figure 4. Temperature dependence of (a) $\Delta \mathrm{A}_{\mathrm{MChD}}$ for 1-(M) and (b) C and A term contributions, and (c) their relative \%, as extracted by two-component multiple regression fits of the experimental data.

In the Barron and Vrbancich theory of $\mathrm{MChD},{ }^{2}$ an absorptionlike band-shape is associated with $\mathrm{B}$ and $\mathrm{C}$ terms, the latter expected when the population of the ground state levels changes, whereas the derivative-like band-shape is associated with A terms, related to the lifting of the degeneracy of ground- or excited state levels due to the magnetic field, as in MCD spectroscopy. ${ }^{25,26}$ The $A$ and $C$ terms contributions are unraveled by spectral multiple regression fit over the whole temperature range (Figure S15) and their temperature dependence reported in Figure 4b and 4c. A 1/T dependence is evidenced for the $\mathrm{C}$ term, whereas the A term is essentially temperature independent, as predicted by the theory. ${ }^{2}$

In conclusion, we have investigated for the first time MChD through NIR light absorption on a chiral lanthanide complex. In this $\mathrm{Yb}^{\mathrm{III}}$ derivative a fine-structured strong MChD signal associated to the ${ }^{2} \mathrm{~F}_{5 / 2} \leftarrow{ }^{2} \mathrm{~F}_{7 / 2}$ transition has been detected. The analysis of the temperature and magnetic field dependence of the MChD spectra has revealed $i$ ) the major role of crystal field splitting and vibronic coupling in defining the overall shape of the MChD spectrum, and ii) the temperature dependence of the MChD lineshape confirms the presence and the relative importance of the socalled A and $\mathrm{C}$ terms of the MChD theory and represents the first unambiguous identification of the underlying mechanisms. Furthermore, this study evidences that ligand-centered helical chirality can be effectively used to enable strong MChD signals at the lanthanide center. This study opens the door for the detection of MChD in chiral lanthanide SMM with an opened hysteresis, enabling the optical read-out of magnetic data with unpolarized light.

\section{ASSOCIATED CONTENT}

\section{Supporting Information}

The Supporting Information is available free of charge at .... Additional figures, tables and experimental section.

\section{AUTHOR INFORMATION}

\section{Corresponding Authors}

matteo.atzori@lncmi.cnrs.fr

jeanne.crassous@univ-rennes1.fr

fabrice.pointillart@univ-rennes1.fr

\section{Notes}

The authors declare no competing financial interests.

\section{ACKNOWLEDGMENT}

The French National Research Agency (ANR) and the European Research Council (ERC) are acknowledged for financial support through MONAFER (ANR-18-CE09-0032), SMMCPL (ANR-19CE29-0012-02) and MaChiNaCo (ANR-19-CE09-0018) projects and the European Union's Horizon 2020 research and innovation program (ERC-CoG MULTIPROSMM, grant agreement No725184). MG and BLG thank the French GENCI/IDRISCINES centers for high-performance computing resources. Dr. Clément Faugeras (LNCMI-CNRS) is kindly acknowledged for Raman spectra collection.

\section{REFERENCES}



Dichroism. Nature 1997, 390, 493-494.

(2) Barron, L. D.; Vrbancich, J. Magneto-Chiral Birefringence and Dichroism. Mol. Phys. 1984, 51, 715-730.

(3) Wagnire, G. H. On Chirality and the Universal Asymmetry; Wiley-VCH Verlag GmbH \& Co. KGaA: Weinheim, Germany, 2007.

(4) Barron, L. D. Chirality, Magnetism and Light. Nature 2000, 405, 895-896.

(5) Galán-Mascarós, J. R. Bring to Light. Nat. Phys. 2015, 11, 7-8.

(6) Ceolín, M.; Goberna-Ferrón, S.; Galán-Mascarós, J. R. Strong Hard X-Ray Magnetochiral Dichroism in Paramagnetic Enantiopure Molecules. Adv. Mater. 2012, 24, 3120-3123.

(7) Atzori, M.; Rikken, G. L. J. A.; Train, C. Magneto-Chiral Dichroism: A Playground for Molecular Chemists. Chem. - A Eur. J. 2020, 26, 9784-9791.

(8) Ishii, K.; Hattori, S.; Kitagawa, Y. Recent Advances in Studies on the Magneto-Chiral Dichroism of Organic Compounds. Photochem. Photobiol. Sci. 2020, 19, 8-19.

(9) Sun, B.; Liu, X.-F.; Li, X.-Y.; Zhang, Y.; Shao, X.; Yang, D.; Zhang, H.-L. Two-Dimensional Perovskite Chiral Ferromagnets. Chem. Mater. 2020, 32, 8914-8920.

(10) Atzori, M.; Breslavetz, I.; Paillot, K.; Inoue, K.; Rikken, G. L. J. A.; Train, C. A Chiral Prussian Blue Analogue Pushes MagnetoChiral Dichroism Limits. J. Am. Chem. Soc. 2019, 141, 2002220025.

(11) Atzori, M.; Santanni, F.; Breslavetz, I.; Paillot, K.; Caneschi, A.; Rikken, G. L. J. A.; Sessoli, R.; Train, C. Magnetic Anisotropy Drives Magnetochiral Dichroism in a Chiral Molecular Helix Probed with Visible Light. J. Am. Chem. Soc. 2020, 142, 13908-13916.

(12) Sessoli, R.; Boulon, M.-E.; Caneschi, A.; Mannini, M.; Poggini, L.; Wilhelm, F.; Rogalev, A. Strong Magneto-Chiral Dichroism in a Paramagnetic Molecular Helix Observed by Hard X-Rays. Nat. Phys. 2015, 11, 69-74.

(13) Guo, F.-S.; Day, B. M.; Chen, Y.-C.; Tong, M.-L.; Mansikkamäki, A.; Layfield, R. A. Magnetic Hysteresis up to 80 Kelvin in a Dysprosium Metallocene Single-Molecule Magnet. Science 2018, 362, 1400-1403.

(14) Goodwin, C. A. P.; Ortu, F.; Reta, D.; Chilton, N. F.; Mills, D. P. Molecular Magnetic Hysteresis at 60 Kelvin in Dysprosocenium. Nature 2017, 548, 439-442.

(15) Train, C.; Gruselle, M.; Verdaguer, M. The Fruitful Introduction of Chirality and Control of Absolute Configurations in Molecular Magnets. Chem. Soc. Rev. 2011, 40, 3297.

(16) Ou-Yang, J.-K.; Saleh, N.; Fernandez Garcia, G.; Norel, L.;
Pointillart, F.; Guizouarn, T.; Cador, O.; Totti, F.; Ouahab, L.; Crassous, J.; Le Guennic B. Improved Slow Magnetic Relaxation in Optically Pure Helicene-Based Dy(III) Single Molecule Magnets. Chem. Commun. 2016, 52, 14474-14477.

(23) Shen, C.; Loas, G.; Srebro-Hooper, M.; Vanthuyne, N.; Toupet, L.; Cador, O.; Paul, F.; López Navarrete, J. T.; Ramírez, F. J.; Nieto-Ortega, B.; Casado, J.; Autschbach, J.; Vallet, M.; Crassous, J. Iron Alkynyl Helicenes: Redox-Triggered Chiroptical Tuning in the IR and Near-IR Spectral Regions and Suitable for Telecommunications Applications. Angew. Chemie Int. Ed. 2016, 55, 8062-8066.

(24) Gendron, F.; Di Pietro, S.; Abad Galán, L.; Riobé, F.; Placide, V.; Guy, L.; Zinna, F.; Di Bari, L.; Bensalah-Ledoux, A.; Guyot, Y.; et al. Luminescence, Chiroptical, Magnetic and Ab Initio Crystal-Field Characterizations of an Enantiopure Helicoidal Yb(III) Complex. Inorg. Chem. Front. 2021, DOI: doi.org/ 10.1039/D0QI01194K.

(25) Mason, W. R. A Practical Guide to Magnetic Circular Dichroism Spectroscopy; 2006.

(26) Stephens, P. J. Theory of Magnetic Circular Dichroism. J. Chem. Phys. 1970, 52, 3489.

\section{TOC Graphic}




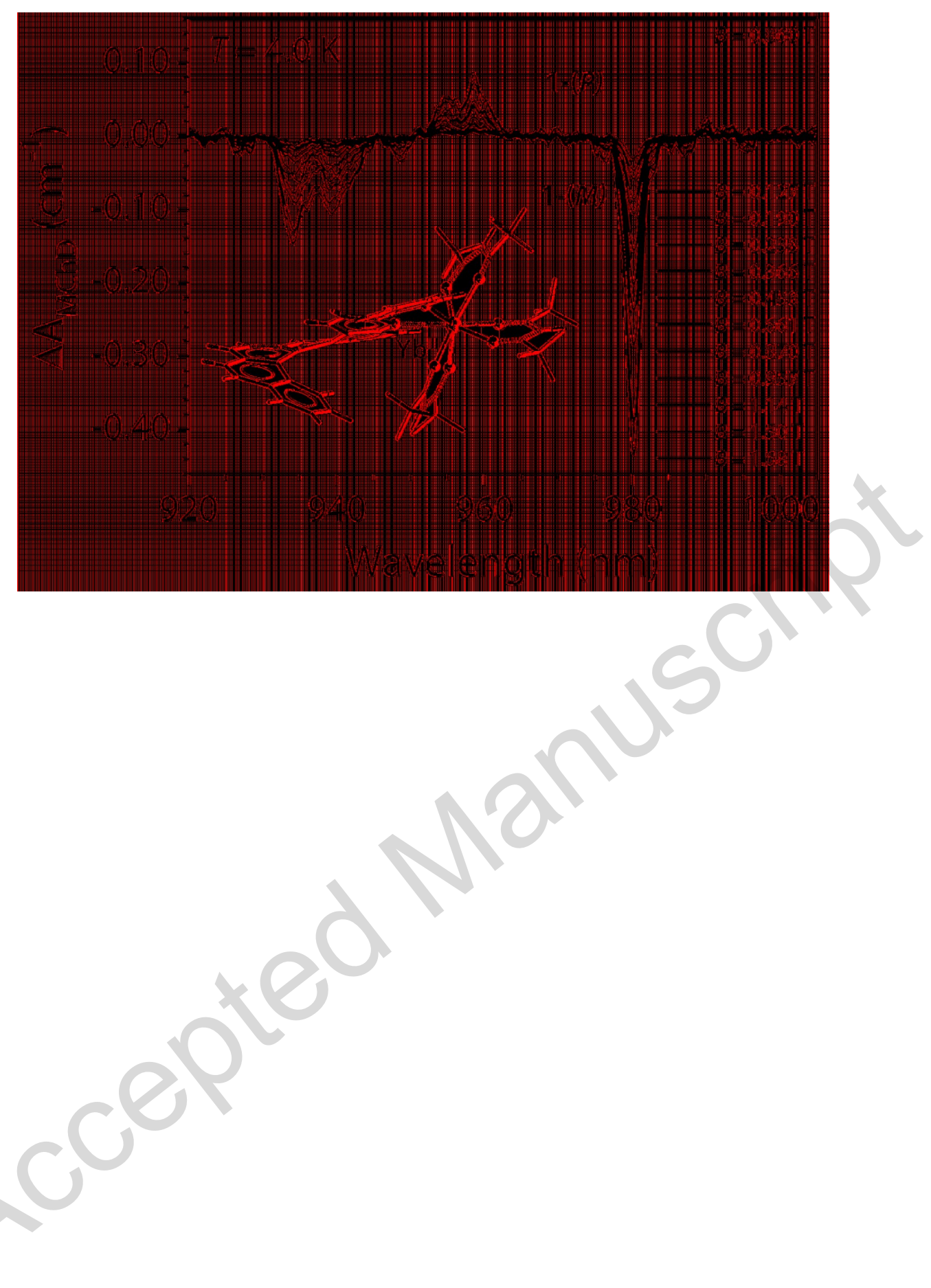

